\title{
Eliminação da solução de ácido clorídrico na limpeza da vidraria utilizada em análise de fósforo total pelo método do ácido ascórbico
}

\section{Elimination of hydrochloric acid solution in glassworks cleaning used in analysis of total phosphorus by acid ascorbic method}

- Data de entrada: $17 / 08 / 2015$

Data de aprovação: $31 / 05 / 2017$

Luis Alcides Schiavo Miranda*/ Carine Helena Molz/Jackson Saint Pierre Dull Ritter/ Luis André Hoff

DOI: $10.4322 /$ dae.2018.007

\section{Resumo}

As análises da concentração de fósforo total em águas servem para avaliar o nível de contaminação destas pela ação humana. Compostos fosforados também estão presentes nos detergentes utilizados na limpeza das vidrarias de laboratório, motivo pela qual estas são posteriormente lavadas com uma solução de ácido clorídrico a quente. 0 presente artigo procura testar e comprovar que o método de lavagem da vidraria para determinação de fósforo com uma solução de ácido clorídrico 3:1 a quente pode ser substituído pela lavagem com uma solução de hipoclorito de sódio a 10\%, em função da toxidade dos vapores de ácido clorídrico gerados na lavagem a quente. A análise de fósforo total é feita pelo método do ácido ascórbico. Este comparativo utilizou métodos estatísticos e demonstrou uma correlação acima de $95 \%$ nos resultados, em uma avaliação de 100 amostras, comparando as duas metodologias de lavagem da vidraria Palavras-chave: Lavagem de Vidrarias. Fósforo Total. Hipoclorito de Sódio.

\section{Abstract}

The analysis of the concentration of total phosphorus in waters is usually used to evaluate the level of contamination by human action. Phosphorus compounds also are components of detergents that clean the glassware laboratory, what requires the materials used in this analysis should be washed with a hot hydrochloric acid solution. The object of this study is to test and prove the efficiency of the replacement of the hot acid wash with a solution of hydrochloric acid by washing with sodium hypochlorite, motivated by the toxic fumes of hydrochloric acid generated in the process. This analysis of total phosphorus is made by the ascorbic acid method. This comparative was done by statistical methods, and demonstrated a correlation of more than 95\% results with an evaluation of 100 samples performed compared with each of the washes. Keywords: Wash glassworks of laboratory. Total Phosphorus. Sodium Hypochlorite.

Luis Alcides Schiavo Miranda - Graduado em Química Industrial. Mestre em Ciência dos Alimentos. Doutor em Ciências com ênfase em Biotecnologia Ambiental (Processos Anaeróbios de Tratamento de Efluentes) e Pós-doutorado em Recursos Hídricos e Saneamento Ambiental. Professor permanente do Programa de Pós-Graduação em Engenharia Civil - Mestrado Acadêmico em Gerenciamento de Resíduos da Unisinos. Carine Helena Molz - Técnico em Química. Graduada em Química Licenciatura. Mestre em Engenharia Civil com Ênfase em Gerenciamento de Resíduos. Trabalha na Companhia Riograndense de Saneamento, no Departamento de Controle de Efluentes Líquidos e Resíduos.

Jackson Saint Pierre Dull Ritter - Técnico em Química. Graduado em Administração de Empresas. Especialização em Gerenciamento Ambiental. Trabalha na Companhia Riogranense de Saneamento - CORSAN como técnico responsável pelo Laboratório Regional de Esgotos do Litoral Norte do RS.

Luis André Hoff - Técnico em Química. Graduado em Gestão Ambiental. Especialização em Saneamento Ambiental. Trabalha na Companhia Riograndense de Saneamento no Departamento de Controle de Efluentes Líquidos e Resíduos.

*Endereço para correspondência: Av. Rudá, 1733, São Jorge Capão da Canoa - RS - CEP 95555000 . E-mail: lalcidesaunisinos.br. 


\section{INTRODUÇÃO}

O fósforo é um dos principais nutrientes para o funcionamento dos processos biológicos, devendo estar presente em relações adequadas entre as frações carbônicas e nitrogenadas. É comumente encontrado nas águas superficiais decorrentes das descargas dos esgotos sanitários tratados e não tratados. A matéria orgânica fecal e os detergentes líquidos e em pó empregados em larga escala doméstica constituem as principais fontes de fósforo nos esgotos domésticos. Alguns efluentes industriais, como os das indústrias de fertilizantes, pesticidas, químicas em geral, conservas alimentícias, abatedouros, frigoríficos e laticínios apresentam em seus efluentes fósforo em quantidades excessivas. As águas drenadas em áreas agrícolas e urbanas podem gerar quantidade excessiva de fósforo nas águas naturais. $O$ fósforo é considerado um parâmetro útil para dimensionar o nível de contaminação em águas naturais e residuárias, pois é um dos responsáveis pelo processo de eutrofização, pelo aumento dos níveis de nutrientes no meio aquático. Conforme Nuvolari et al. (2003), o fósforo apresenta-se nas formas de ortofosfatos, polifosfatos e fosfatos orgânicos, e é o elemento mais visado quando se pretende combater a eutrofização.

Orhon \& Artan (1994) informam que o fósforo pode se apresentar nas águas sob três formas diferentes: os fosfatos orgânicos, que são a forma em que o fósforo compõe moléculas orgânicas, como a de um detergente; os ortofosfatos, que são representados pelos radicais que se combinam com cátions formando sais inorgânicos nas águas; e os polifosfatos ou fosfatos condensados, que são os polímeros de ortofosfatos.

A análise de determinação de fósforo total utilizada neste artigo é o método do Ácido Ascórbico, referência 4500-P E do Standart Methods for
Examination of Water and Wastewater. O princípio do método consiste na reação, em meio ácido, do Molibdato de Amônio e do Tartarato de Antinônio e Potássio com os ortofosfatos, formando um heteropoliácido, o ácido fosfomolibdico, que é reduzido pelo Ácido Ascórbico formando uma coloração azul intensa do Molibdato. Essa coloração é lida em absorbância, utilizando-se um espectofotômetro de feixe simples no comprimento de onda de $880 \mathrm{~nm}$, com uma cubeta de caminho ótico de $25 \mathrm{~mm}$. A concentração de fósforo total é calculada a partir da fórmula da curva de calibração, e o resultado, aplicado em uma curva previamente preparada com padrões de concentrações conhecidas.

Os detergentes utilizados para a limpeza da vidraria na análise contêm em maior ou menor grau fosfatos em diversas formas. Existem detergentes livres de fosfatos, porém estes possuem um custo muito elevado, e por isso são pouco utilizados para as referidas lavagens. A fim de eliminar a contaminação na vidraria é realizada uma lavagem com ácido clorídrico diluído (3:1) a quente. Conforme o International Program on Chemical Safety, vapores de ácido clorídrico são extremamente irritantes para o trato respiratório, podem causar laringite, edema da glote, edema pulmonar, bronquite e até mesmo a morte. Causam ainda desgastes dos dentes, tornando-os amarelados, amolecidos, desgastados e podendo até mesmo quebrar. Devido a todos esses fatores citados anteriormente, para a realização desta limpeza é necessária a utilização de capela de exaustão, liberando estes vapores para o meio ambiente, além do risco de queimaduras e corrosão de equipamentos como a chapa elétrica.

O objetivo principal deste trabalho é avaliar a eficiência da substituição do ácido clorídrico a quente pelo hipoclorito de sódio a frio na lavagem de vidraria. 


\section{MATERIAIS E MÉTODOS}

A lavagem ácida é feita após a lavagem com detergente comercial para higienização da vidraria. 0 ácido clorídrico diluído é adicionado a frio aos erlenmeyers e estes são aquecidos em chapa elétrica até a ebulição. Após o resfriamento, os erlenmeyers são lavados em água corrente e depois com água destilada. Na lavagem proposta no teste, a vidraria é somente enxaguada sem a adição em banho de detergente, e colocada imersa em banho de hipoclorito de sódio a $10 \% \mathrm{v} / \mathrm{v}$ por aproximadamente 4 horas. 0 material é posteriormente enxaguado com água corrente e água destilada.

Para avaliação da eficiência do método, foram realizadas 100 determinações em duplicata do parâmetro fósforo total em amostras de efluentes pelo método do ácido ascórbico, sendo uma com a vidraria lavada conforme o método Standart (ácido clorídrico diluído a quente) e a sua duplicata com a vidraria lavada com o método proposto (hipoclorito de sódio). Todo o restante do procedimento analítico foi feito de forma idêntica e em mesma data para ambas as lavagens.

Optou-se ainda por utilizar amostras reais, e não padrões de fósforo para que os eventuais interferentes, como os arsenatos, pudessem ser desconsiderados na utilização prática desse novo procedimento. Estes podem reagir com o molibdato de amônio produzindo coloração azul, que eventualmente poderá interferir no resultado.

Os resultados foram comparados pelos métodos estatísticos ANOVA e análise da variância. Elliot and Woodward (2006) definem que o método ANOVA é, de maneira geral, uma extensão do teste t-student emparelhado, que pode ser usado para medidas repetidas. $O$ teste é reconhecido por comparar a variância entre dois ou mais grupos de resultados e verificar se os resultados são significativamente diferentes. 0 teste considera uma variância média estatística entre os conjuntos de dados menor que $5 \%$ como não significativamente diferentes, podendo validar a eficiência da nova lavagem em análise. $O$ teste $F$ compara igualmente se os resultados podem ser considerados semeIhantes, mas com a restrição de trabalhar somente com dois conjuntos de dados. Como estamos testando somente estes dois grupos (lavagem com ácido clorídrico X lavagem com hipoclorito de sódio), este último pode ser utilizado para reforçar os estudos estatísticos.

\section{DISCUSSÃO DE RESULTADOS}

Os resultados das 100 amostras realizadas em paralelo, uma com cada método de lavagem, posteriormente aplicadas à análise estatística ANOVA estão apresentado na Tabela 1.

Tabela 1:Teste ANOVA realizado entre a lavagem Ácida e Hipoclorito.

\begin{tabular}{|l|c|c|c|c|c|c|}
\hline $\begin{array}{l}\text { Fonte da } \\
\text { variação }\end{array}$ & $\begin{array}{c}\text { SQ } \\
\text { (Soma dos } \\
\text { Quadrados) }\end{array}$ & $\begin{array}{c}\text { gl } \\
\text { (Graus de } \\
\text { Liberdade) }\end{array}$ & $\begin{array}{c}\text { MQ } \\
\text { (Média } \\
\text { Quadrada) }\end{array}$ & F calc & p-valor & F crítico \\
\hline Entre grupos & 0,015842 & 1 & 0,015842 & 0,001605 & 0,968084 & 3,888853 \\
\hline $\begin{array}{l}\text { Dentro dos } \\
\text { grupos }\end{array}$ & 1954,395 & 198 & 9,870680343 & - & - & - \\
\hline Total & 1954,411 & 199 & - & - & - & - \\
\hline
\end{tabular}


O estudo estatístico dos dados retornou um "F crítico" calculado, que é o máximo valor de referência da variância das amostras dividido pela variância entre as amostras, que pode ser atingido considerando os $5 \%$ de variância entre os resultados obtidos de 3,888853. A hipótetse testada pode ser aceita, pois o $\mathrm{F}$ calculado, que deve ser menor que o $\mathrm{F}$ crítico para aprovar a tese, foi atingido com um valor de 0,001605. Como reitera Cadima, J. e Silva, P. (2008) quando F Calculado < F Crítico não há variação significativa entre os resultados. Para confirmar essa análise, podemos verificar que o valor de $P>0$ indica que os valores também são aprovados na probabilidade condicional para análise estatística com o nível de significância escolhido de $95 \%(\alpha=0,05)$.

A mesma análise estatística comparativa entre os dois grupos de resultados foi avaliada por meio do teste $F$, obtendo os resultados apresentados na tabela 2 .
Tabela 2: Teste F comparando os resultados com Lavagem Ácida X Lavagem Hipoclorito.

\begin{tabular}{|l|c|c|}
\hline Teste F & Lavagem Ácida & Lavagem Hipoclorito \\
\hline Média & 2,8914 & 2,8736 \\
\hline Variância & 10,00032 & 9,741041 \\
\hline Observações & 100 & 100 \\
\hline Graus de liberdade & 99 & 99 \\
\hline & Análise da Variância \\
\hline F & \multicolumn{2}{|c|}{1,026617} \\
\hline P(F<=f) uni-caudal & 0,448143 \\
\hline Fcrítico uni-caudal & \multicolumn{2}{|c|}{1,394061} \\
\hline
\end{tabular}

Neste caso, o valor de $\mathrm{P}<\mathrm{F}$ crítico demonstra que não há variação significativa entre os resultados, comprovando a hipótese, sendo que, neste caso, o grau de significância adotada é também de 95\%.

Os resultados foram plotados comparando os valores obtidos em cada lavagem, para avaliar os dados em diversas concentrações de fósforo.

A Figura 1 representa esse comparativo gráfico entre os resultados de concentração em $\mathrm{mg} / \mathrm{L}$ de fósforo obtidos nas lavagens com ácido clorídrico e hipoclorito de sódio, e demonstra que são significativamente semelhantes em todas as faixas de concentração analisadas no presente estudo.

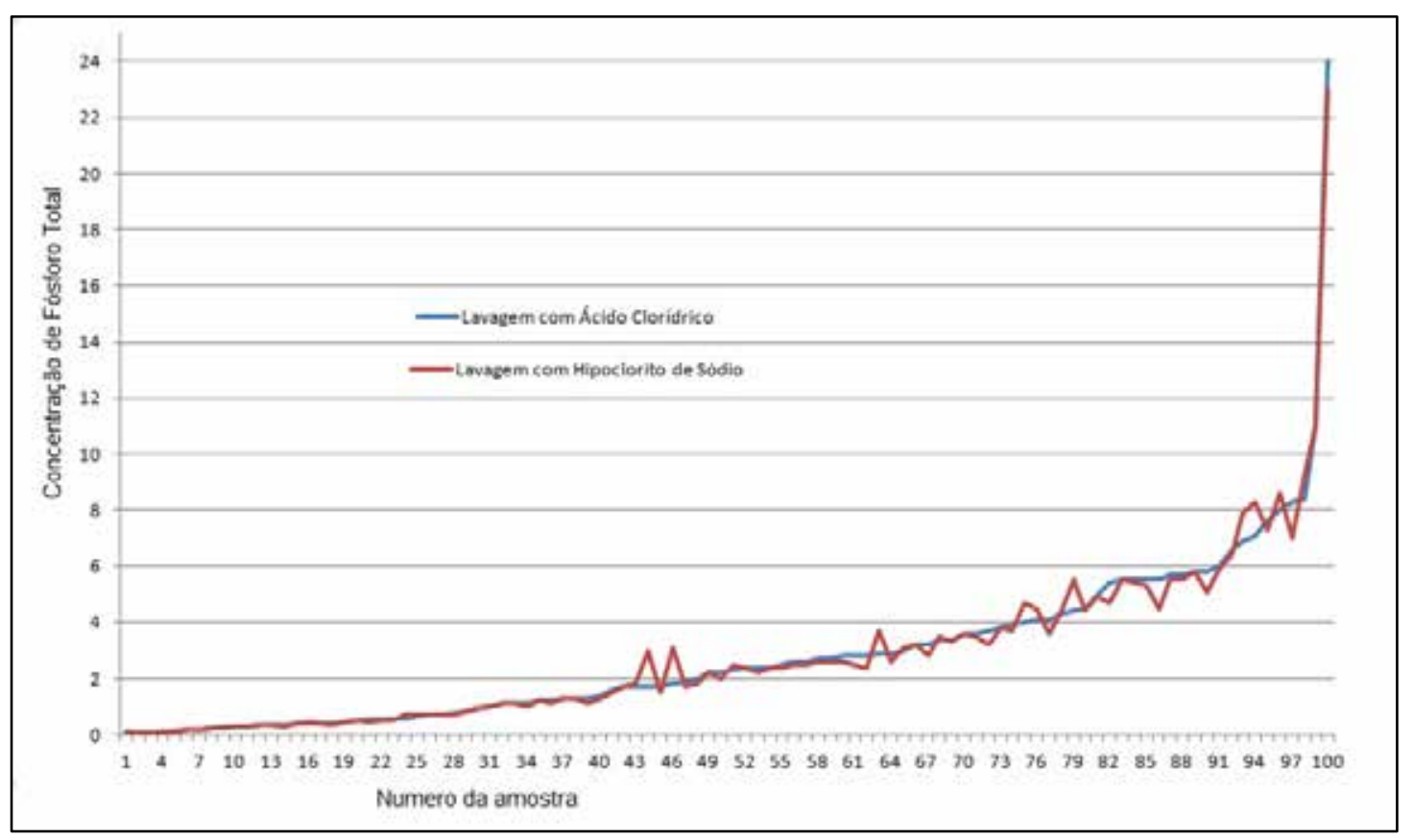

Figura 1: Comparativo dos resultados de P determinados, após as lavagens ácida e hipoclorito de sódio das vidrarias. 
A determinação de fósforo total pelo método do ácido ascórbico é feito por uma leitura em espectrofotômetro que abrange uma concentração de fósforo de 0,0 até $2,0 \mathrm{mg} / \mathrm{L}$, sendo todas as de- mais concentrações oriundas de diluições das amostras a fim de enquadrar o resultado nesta faixa. Na figura 2, avaliam-se somente as amostras com leitura direta do método, sem diluições.

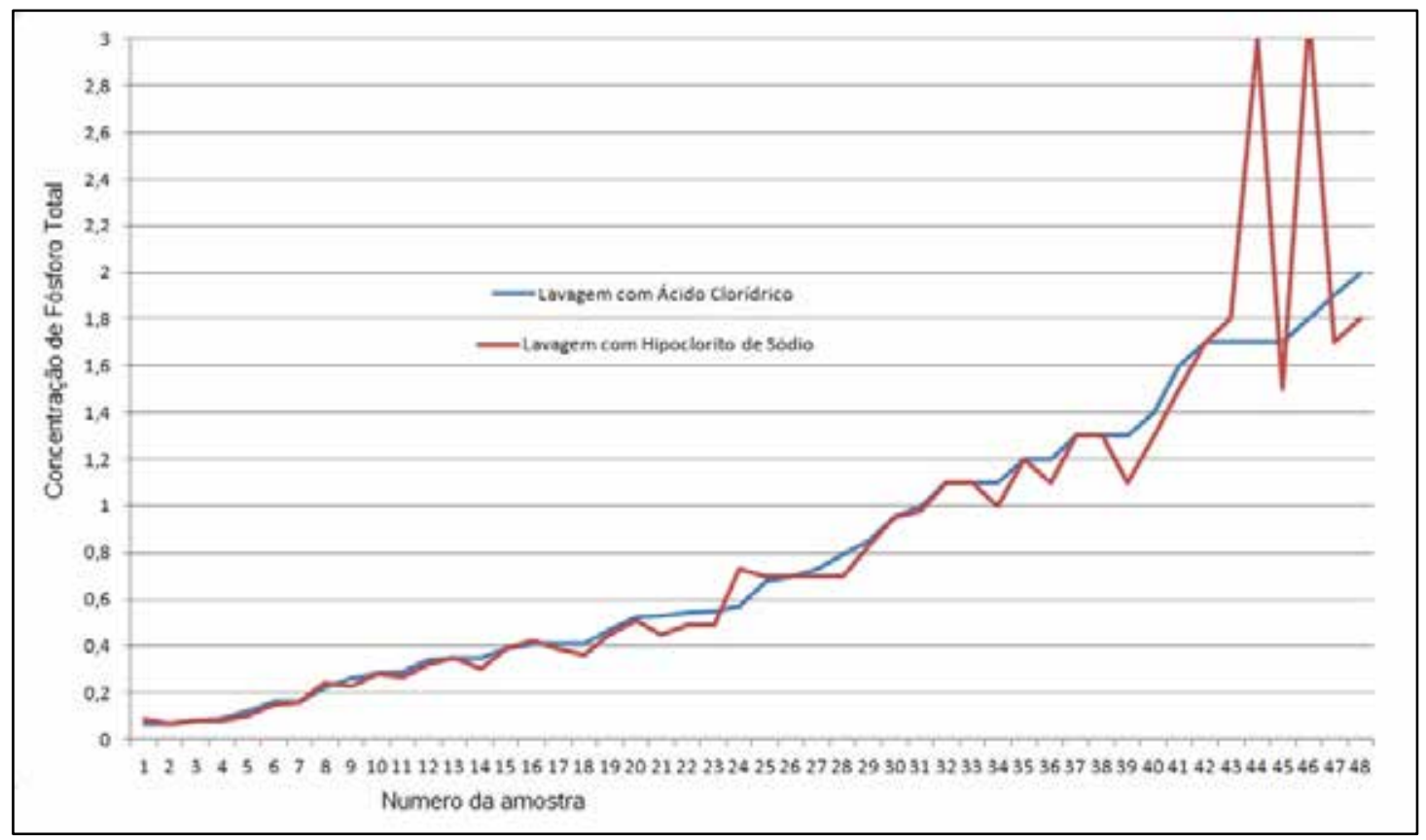

Figura 2: Comparativo gráfico dos resutados entre lavagem ácida e lavagem com hipoclorito com valores inferiores a $2,0 \mathrm{mg} / \mathrm{L}$ de $P$.

\section{CONCLUSÃO}

Os dados observados estatisticamente permitem concluir que não há diferença significativa entre os resultados, podendo ser utilizado um ou outro método de lavagem das vidrarias sem impacto na confiabilidade dos resultados.

\section{REFERÊNCIAS}

NUVOLARI ET AL, Esgoto Sanitário, coleta, transporte, tratamento e reúso agrícola, São Paulo: Ed Edgar Blücher, 2003.

SPERLING M. V. , Lodos Ativados, Belo Horizonte: UFMG, 2002, $2^{\mathrm{a}}$ edição.

CADIMA, J. E SILVA, P., Matemática e Estatística - Análise de Variância (ANOVA), DM/ISA - Lisboa - Portugal, 2008-09.
[IPCS-INCHEM] International Program on Chemical Safety. Disponível em: http://www.inchem.org. Acesso em Novembro de 2012.

ELLIOT, A. AND WOODWARD, W. 2006. Statistical Analysis Quick Reference Guidebook. United States: Sage.

FIDÉLIS, G.C>. Metrologia, Técnicas para Assegurar Medições Confiáveis. Florianópolis, SC, 2010. $1^{\circ}$ Edição, CECT.

\section{BIBLIOGRAFIA CONSULTADA}

Standard Methods for the Examination of Water and Wastewater, $22^{\text {nd }}$ Edition, 2012 Fósforo total, Variáveis Químicas, CETESB, São Paulo.

ALARIE, Y. Dose-Response Analysis in Animal Studies: Prediction of Human Responses, USA, The National Institute of Environmental Health Sciences (NIEHS),Vol. 42, 1981.

EURACHEM. The Fitness for Purpose of Analytical Methods A Laboratory Guide to Method Validation and Related Topics, 1998 http://www.est.ufpr.br/ce003/material/cap7.pdf 